\title{
Traffic Road Sign Detection and Recognition for Automotive Vehicles
}

\author{
Md. Safaet Hossain \\ Department of Electrical Engineering and \\ Computer Science North South University, Dhaka \\ Bangladesh
}

\author{
Zakir Hyder \\ Department of Electrical Engineering and \\ Computer Science North South University, Dhaka \\ Bangladesh
}

\begin{abstract}
Traffic road sign detection and recognition is important to transport system with a robotic eyes or camera while driving in the road. This paper presents and overview the traffic road sign detection and recognition, we developed and implemented the procedure to extract the road sign from a natural complex image. The main objective of this paper is to design and construct a computer based system which can automatically detect the direction of the road sign. This paper is based upon a major approach to detect the direction. In this paper, we will demonstrate the basic idea of how detect the area and extract it. This system will play an important role for the detection purpose of specific domains like island, schools, traffic sign, universities, hospitals, offices etc.
\end{abstract}

\section{Keywords}

Web based applicatoin testing, performance testing, functional testing, test methods integration, e-commece.

\section{INTRODUCTION}

The sign which is placed at the side of roads to impart information to road users is known as road signs or traffic signs. There are four types of traffic signs that are shown in the traffic code: a) warning; b) prohibition; c) obligation; and d) informative. Depending on the form and the color, the warning signs are equilateral triangles with one vertex upwards. They have a white background and are surrounded by a red border. Prohibition signs are circles with a white or blue background and a red border. Both warning signs and prohibition signs have a yellow background if they are located in an area where there are public works. To indicate obligation, the signs are circles with a blue background. Informative signs have the same color. Finally, there are two exceptions: a) the yield sign, an inverted triangle; and the stop sign, a hexagon. To detect the position of the sign in the image, we must know the two properties i.e., color and shape.

The applications and the difficulty of road sign detection make road sign detection an interesting problem. In terms of applications, road sign detection is quite important for the road sign recognition problem, since it is the most important step for a road sign recognition system. So far, the researchers have mainly focused on the road sign recognition problem, in which the task of finding road sign in an arbitrary background is usually avoided by either manual segmentation of the input image, or by capturing faces against a known uniform background. In the last decade, road sign detection has attracted great attention, as road sign recognition system requires automatic road sign detection as a first step, especially for images with cluttered background. Road sign detection also has potential applications in human computer interface and surveillance systems. Road sign detection is difficult due to three main reasons. First, there is a large component of non-rigidity and textural differences among road sign. Second, road sign detection is also made difficult because of additional features, such as dust, which can either be present or totally absent from a road sign. All these additional features increase the variability of the road sign patterns that a road sign detection system should handle. Third, the presence of unpredictable imaging conditions in an unconstrained environment increases the difficulty of the task. A change in light source distribution can cause a significant change in the appearance of the road sign image. All these things should be taken into consideration when designing a road sign detection system.

\section{METHODOLOGY}

Real time road sign detection system has been developed by sequence of operations. To achieve a successful road sign detection system we use following methodologies:

- Studying literature on different road sign detection methods and image processing.

- $\quad$ Studying the existing method for road sign detection.

- Analyze and design for the proposed system.

- Implement the proposed design of the road sign detection.

- Carrying out experiment and evaluate the system.

The performance of the system has been tested and achieved its desired goal. It was captured by a digital camera and able to detect road sign properly with in a natural image.

\section{RELATED WORK}

There are many researches in the literature deal with Road Sign Recognition (RSR) problem. In this section, we will explore some of those approaches.

\subsection{Fast grey scale road sign model matching and recognition}

Mobile Mapping is a standard technique for compiling cartographic information from a mobile vehicle. [1] author paper proposes a novel method for modeling the recognition in a Mobile Mapping process that consists in fitting a model to recover the sign distortion and applying recognition techniques on weak classifiers cascade results. High variance of sign appearance has made the detection and recognition of road signs a computer vision problem over which many studies have lately been performed. There are two main approaches in this field, the color-based and the grayscalebased sign recognition. Color-based approach allows reducing 
false positives results in the recognition process whereas grayscale methods concentrate on the geometry of the model to recognize it. The color-based studies are based on segmentation by thresholding in color space [2][3][4]. Ghica [5] study focused exclusively on neural networks that are used for image filtering and sign recognition, while other approaches are based on genetic algorithms [6]. The studies on gray-scale images use geometric reasoning [7][8] and most of them on the Hough transforms, and usually color is used as a complementary technique to eliminate false positive results of the classification method.

\subsection{Detection by Adaboost}

The Adaboost algorithm presents a general framework to combine classifiers in order to solve the supervised pattern recognition problem [9]. This approach consists of a) choosing a (weak) classifier, b) modifying example weights in order to give priority to examples where the previous classifiers fail, and c) combining classifiers in a multiple classifier. As a result, each state of the boosting process, which selects a new weak classifier, can be viewed as a feature selection process where features correspond to the image pixels values [10]. The input images of our recognition procedure are provided by the weak classifiers cascade detection process [11].

\subsection{Road Traffic Sign Detection and Classification}

[12] author suggest for road vehicles can have three main roles: a) road detection; b) obstacle detection; and c) sign recognition. The first two have been studied for many years and with many good results, but traffic sign recognition is a less-studied field. Traffic signs provide drivers with very valuable information about the road, in order to make driving safer and easier. They think that traffic signs must play the same role for autonomous vehicles. They are designed to be easily recognized by human drivers mainly because their color and shapes are very different from natural environments. The algorithm described in this paper takes advantage of these features. It has two main parts. The first one, for the detection, uses color thresholding to segment the image and shape analysis to detect the signs. The second one, for the classification, uses a neural network. Some results from natural scenes are shown. On the other hand, the algorithm is valid to detect other kinds of marks that would tell the mobile robot to perform some task at that place.

\subsection{Traffic sign detection}

There are four types of traffic signs that are shown in the traffic code: a) warning; b) prohibition; c) obligation; and d) informative. Depending on the format of the color, the warning signs are equilateral triangles with one vertex upwards. They have a white background and are surrounded by a red border. Prohibition signs are circles with a white or blue background and a red border. Both warning signs and prohibition signs have a yellow background if they are located in an area where there are public works. To indicate obligation, the signs are circles with a blue background. Informative signs have the same color. Finally, there are two exceptions: a) the yield sign, an inverted triangle; and b) the stop sign, a hexagon. They were not studied here. To detect the position of the sign in the image, we must know the two properties we talked about before, i.e., color and shape.

\subsection{Color Thresholding}

The most intuitive color space is the RGB system. The color of every pixel is defined by three components: red, green, and blue. Because of this, the color threshold has the following expression:

$$
g(x, y)=k_{l}\left\{\begin{array}{l}
R_{a} \leq f_{r}(x, y) \leq G_{b} \\
G_{a} \leq f_{g}(x, y) \leq G_{b} \\
B_{a} \leq f_{b}(x, y) \leq G_{b}
\end{array}\right.
$$

$g(x, y)=k_{2}$ in any other case

where $f_{r}(x, y), f_{g}(x, y)$ and $f_{b}(x, y)$ are, respectively, the functions that give the red, green, and blue levels of each point of the image. One of the greatest inconveniences of the previous color space is that it is very sensitive to lighting changes.

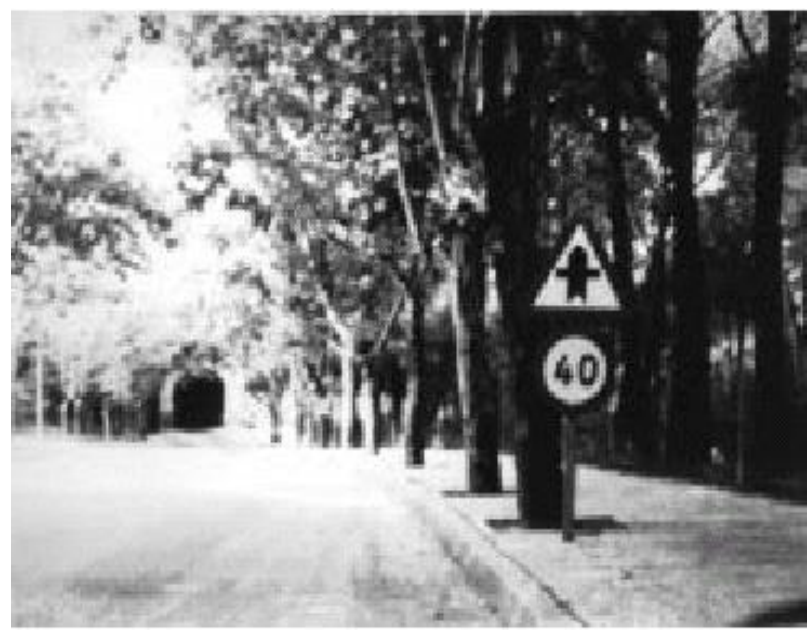

(a)

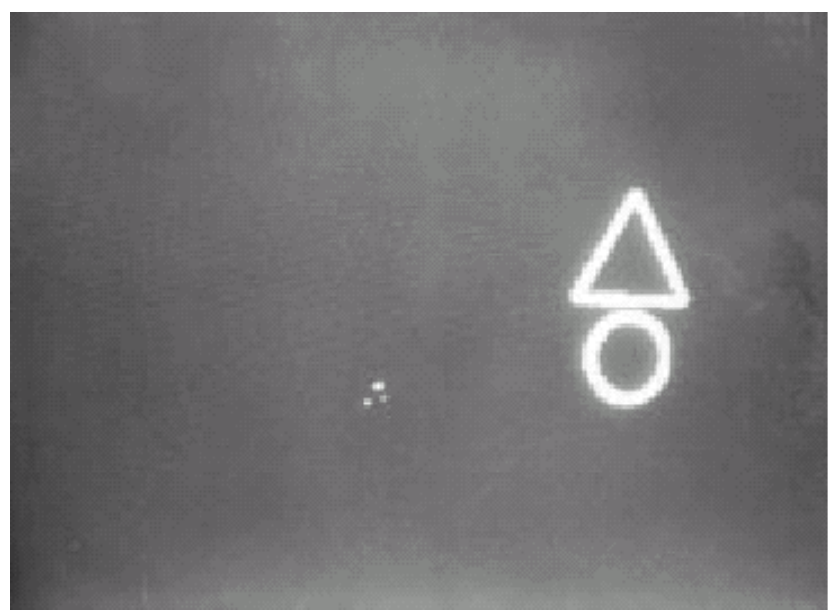

(b)

Figure 1: (a) Color threshold (b) Original image

\subsection{Experimental Results}

In this section, we will present some results for the red (prohibition) signs. The first row of Figure 2.9 shows samples of road images. The second row shows the results of the Bayes classifier on the road images set. The third row shows the gray level labels for the red color in the captured images. The last row shows the matching results. 


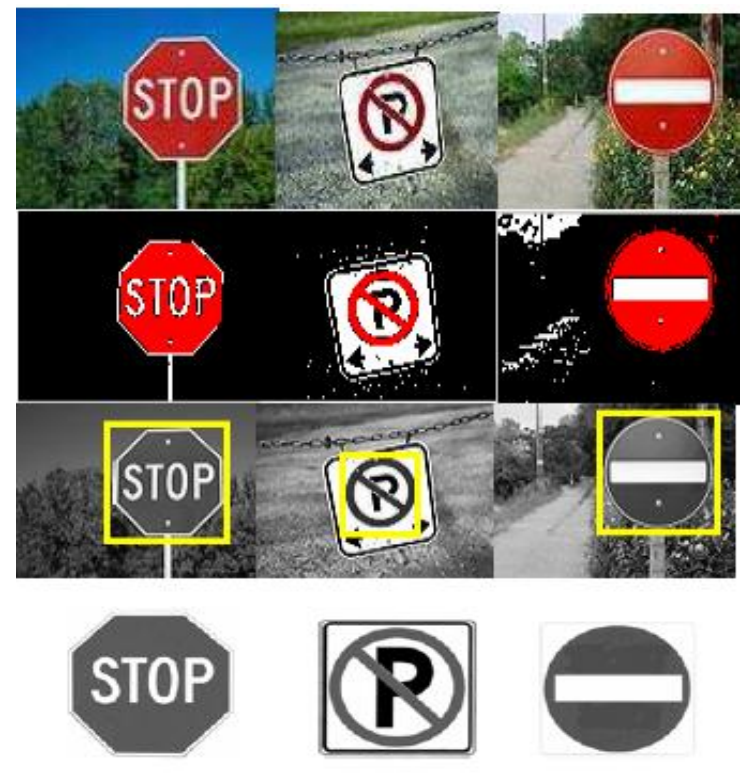

Figure 2: Sample results

\section{SYSTEM ARCHITECTURE}

Following figure shows the system architecture

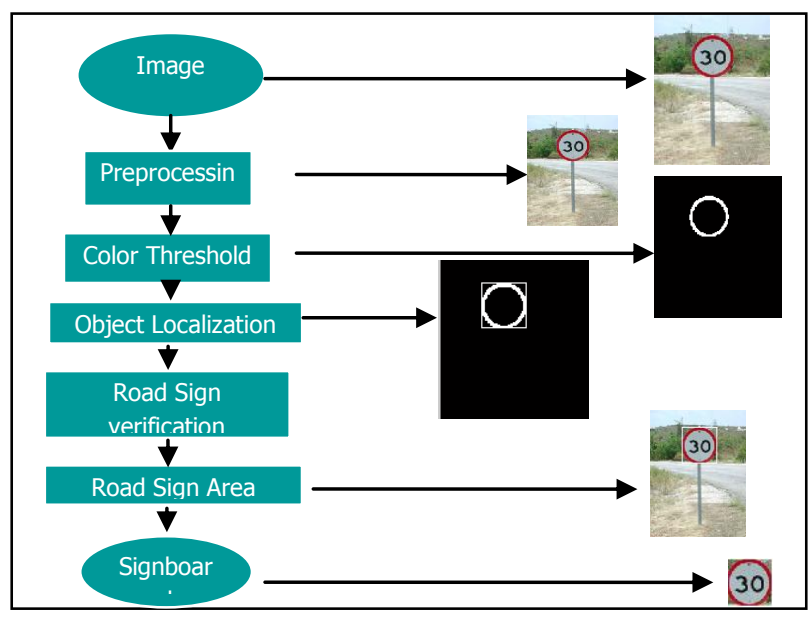

Figure 3: System Architecture

\subsection{Experimental Result}

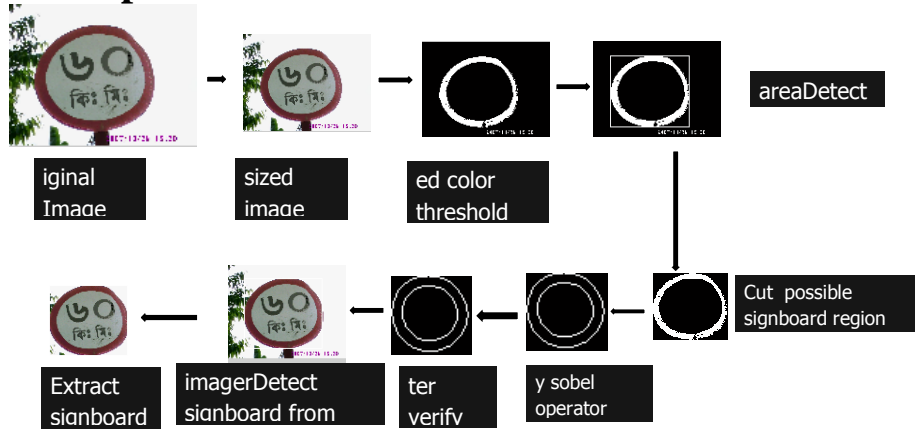

Figure 4: Experimental results with sign detection

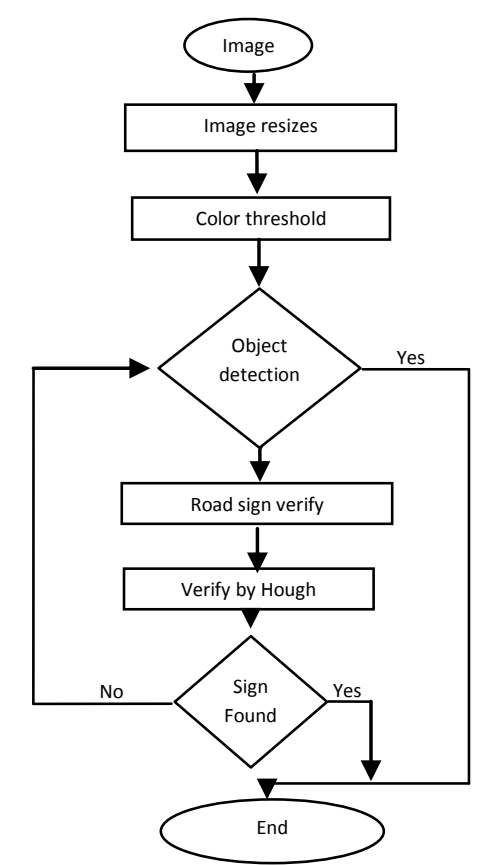

Figure 5: System Flow chart

This paper deals with object detection in outdoor an environment which is usefulness for Driver Support systems and Intelligent Autonomous Vehicles to take some decisions about their speed, trajectory and send a warning signal indicating over speed, warn or limit illegal ma-oeuvres.

\subsection{Breadth first search}

Bredth first search (BFS) is a searching technique that searches all the space area to locate the region of interest. This technique is widely used for searching. In this project we use to find the problem space where the destination object is road sign.
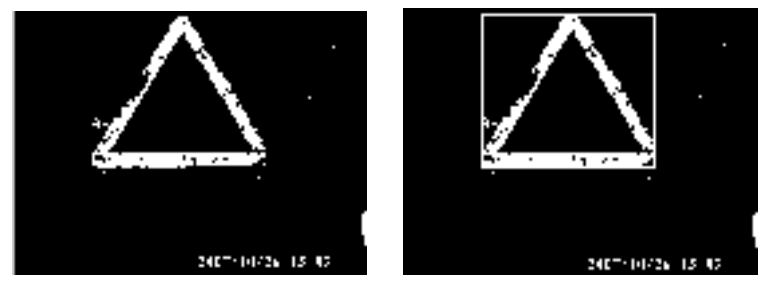

(a)

Figure 6: Object detection (a) Threshold image
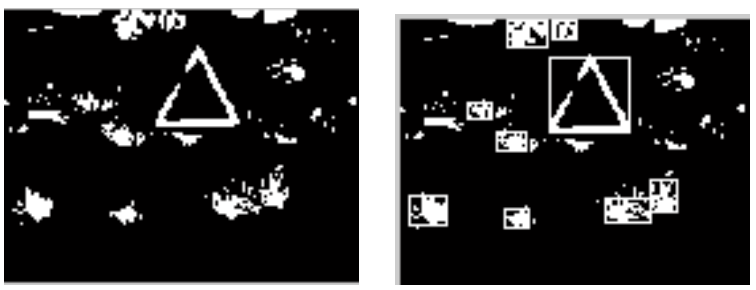

(b)

Figure 7: Object detection (b) detect by BFS 


\subsection{Edge detection}

Where intensity change abruptly in an image is called edge. In edge detection technique the longest connected component are determine by various operator. In this project work we use sobel operator for detecting edges.

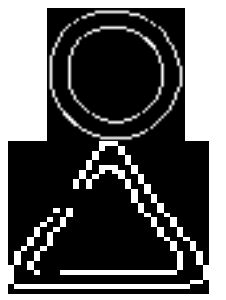

(b)

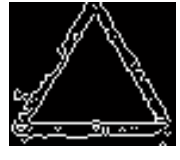

(c)

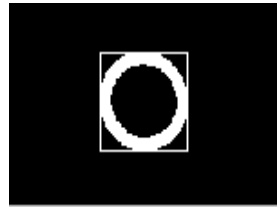

(d)
Figure 8: Edge detection

\subsection{Extract our desired road sign}

After verify the detected sign by applying sobel edge detection operator we extract the road sign from background.
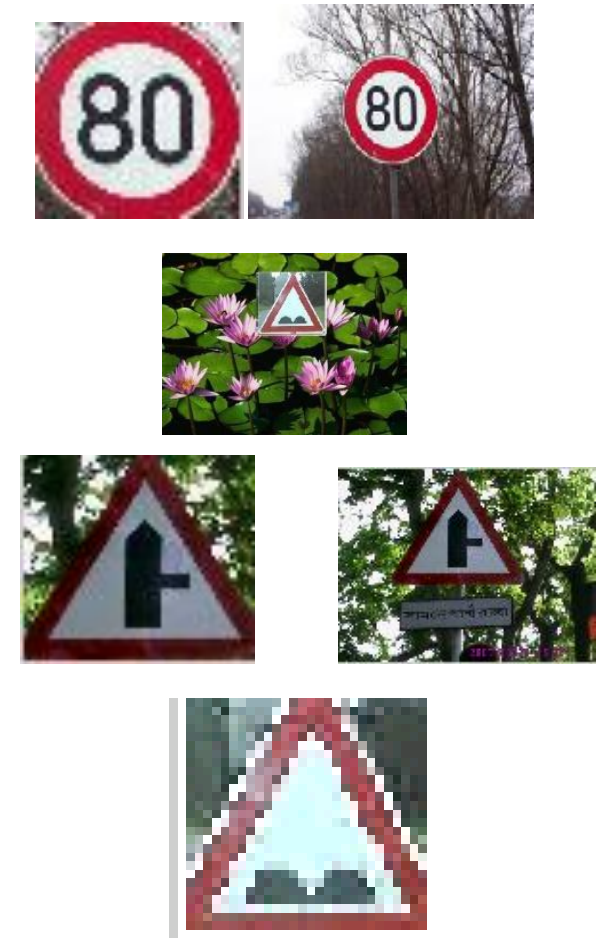

Figure 9: road sign extracting (a) Original image (b) Detected portion

Table 1: Summary of the Result

\begin{tabular}{|l|l|l|l|}
\hline Captured in & $\begin{array}{l}\text { No. of } \\
\text { Image }\end{array}$ & $\begin{array}{l}\text { Correct } \\
\text { result }\end{array}$ & Error result \\
\hline Morning & 50 & $85 \%$ & $15 \%$ \\
\hline Noon & 50 & $90 \%$ & $10 \%$ \\
\hline Evening & 50 & $82 \%$ & $18 \%$ \\
\hline Night & 50 & $50 \%$ & $50 \%$ \\
\hline Total & 200 & $76.75 \%$ & $23.25 \%$ \\
\hline
\end{tabular}

4.5 Sample result

Experiment no 1

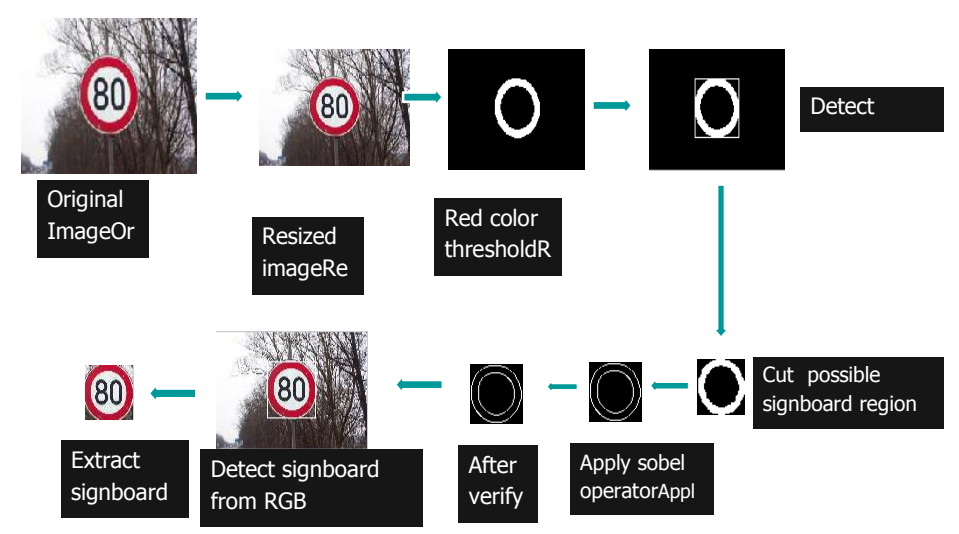

Figure 10: Experiment result no 1

Experiment no 2:

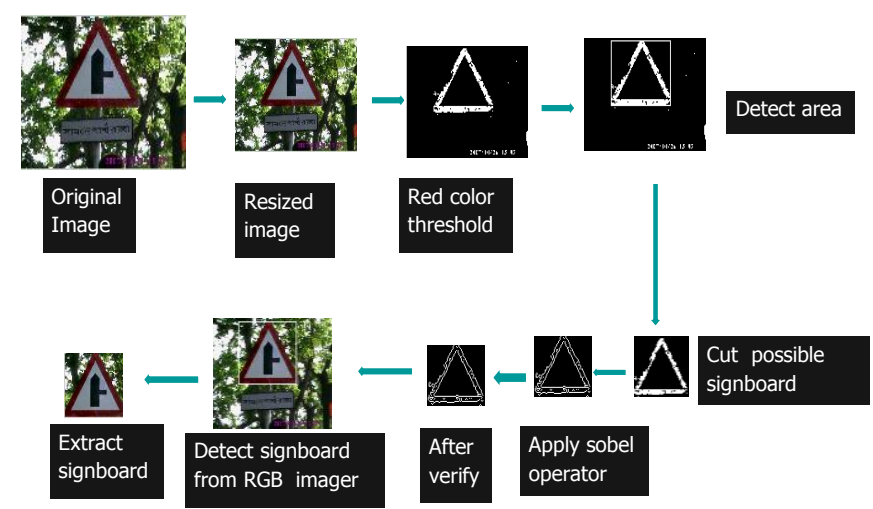

Figure 11: Experiment result no 2

Experiment no 4:

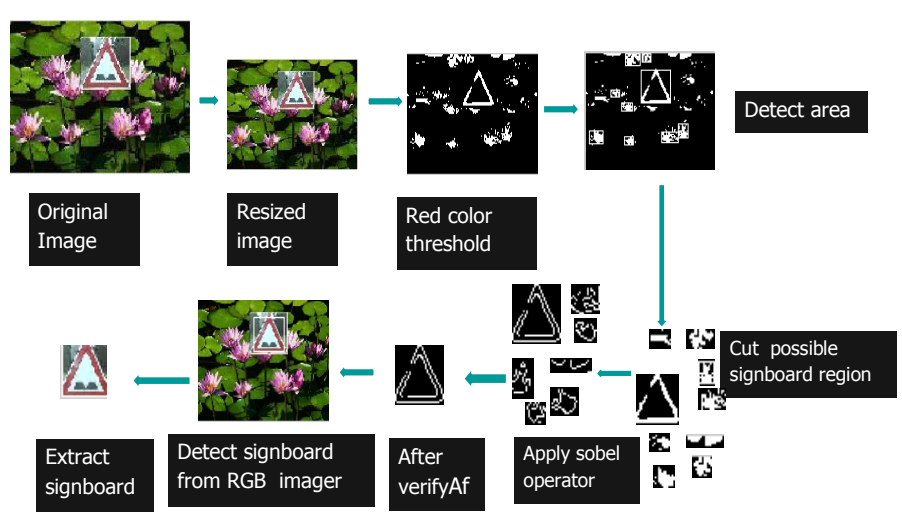

Figure 12: Experiment result no 3 
Experiment no 3:

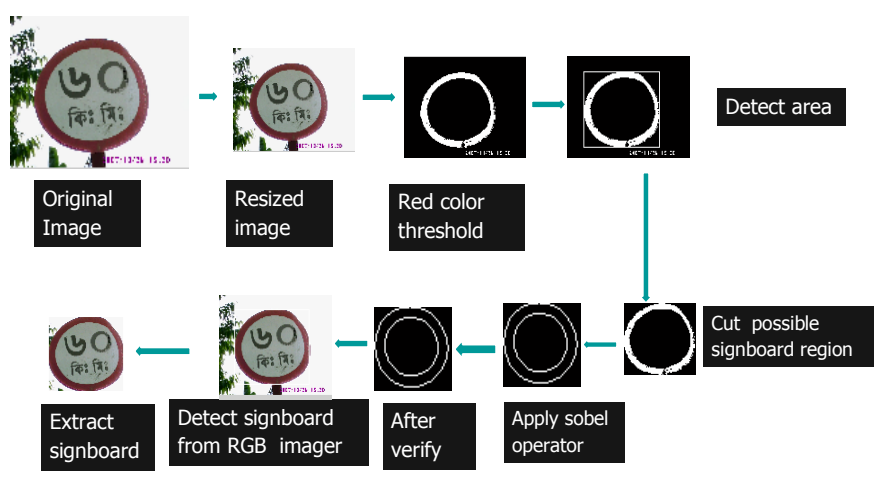

Figure 10: Experiment result no 4

\section{CONCLUSION}

This paper deals with object detection in outdoor environments which are usefulness for Driver Support systems and Intelligent Autonomous Vehicles to take some decisions about their speed, trajectory and send a warning signal indicating over speed, warn or limit illegal ma-oeuvres. It works only red object detection. This technique is deferent for detecting road sign. It helps researchers because it is time effective one.

\section{FUTURE WORK}

Automatic Signboard Recognition using Neural Network All colors Signboard Recognition and Text and symbol recognition and Automatic speed warning.

\section{REFERENCES}

[1] Sergio Escalera and Petia Radeva Centre de Visió per ComputadorEdifici O - Campus UAB, 08193 Bellaterra, Barcelona, Catalonia, Spain \{sescalera,petia\}@cvc.uab.es.

[2] D. Kellmeyer and H. Zwahlen. "Detection of highway warning signs in natural video images using color image processing and neural networks." In IEEE Proc.Int.Conf. Neural Networks 1994, volume 7, pages 4226-4231, 1994.

[3] M. de Saint Blancard. "Road sign recognition: A study of vision-based decision making for road environment recognition." In Vision-based Vehicle Guidance, Springer Series in Perception Engineering. SpringerVerlag, 1992.

[4] Arturo de la Escalera, Luis E. Moreno, Miguel Ángel Salichs and José María Armingol "Road Traffic Signs Detection and Classification," CICYT Project TAP940711-C03-02, jul. 1996.

[5] R. Ghica, S. Lu, and X. Yuan. "Recognition of traffic signs using a multilayer neural network," In Proc. Can Conf. on Electrical and Computer Engineering, 1994.

[6] A. de la Escalera, J.M ${ }^{\mathrm{a}}$ Armingol, M.A. Salich, "TRAFFIC SIGN DETECTION FOR DRIVER SUPPORT SYSTEMS," Systems Enginnering and Automation Division, Universidad Carlos III de Madrid, Leganés, Madrid, Spain.

[7] G. Piccioli, E. D. Michelli, and M. Campani. "A robust method for road sign detection and recognition." In Proc. European Conference on Computer Vision 1994, pages 495-500, 1994.
[8] G. Piccioli, E. D. Michelli, P. Parodi, and M. Campani. "Robust road sign detection and recognition from image sequences.” In Proc. Intelligent Vehicles'94, pages 278283, 1994.

[9] Yoav Freund and Robert E. Schapire. "A decisiontheoretic generalization online learning and an application to boosting," Computational Learning Theory: Eurocolt '95, pages 23-37. Springer-Verlag, 1995.

[10] Paul Viola and Michael J. Jones, "Robust Real-time Object Detection," Cambridge Research Laboratory, Technical Report Series, CRL 2001/01. Feb. 2001.

[11] Jordi Vitrià and Xavier Baró, "Traffic Sign Detection on Greyscale image,” Computer Center Vision, Spain.

[12] Arturo de la Escalera, Member, IEEE, Luis E. Moreno, Member, IEEE, Miguel Angel Salichs, Member, IEEE, and Jos'e Mar'i a Armingol

[13] Andrew Ditzgibbon, Maurizio Pilu, and Robert B. Fisher, "Direct Least Square Fitting of Ellipses," Tern Analysis and Machine Intelligence, vol. 21, no. 5, may. 1999.

[14] R. Brunelli and T. Poggio, "Face Recognition: Features vs. Templates," IEEE Trans. Pattern Analysis and Machine Intelligence, vol. 15, no. 10, pp. 1,042- 1,053, oct. 1993.

[15] Peter N. Belhumeur, Joao P. Hespanha, and David J. Kriegman, "Eigenfaces vs. Fisherfaces: Recognition Using Specific Linear Projection," IEEE Transactions on Pattern Analysis and Machine Intelligence, vol. 19, no. 7, jul. 1997.

[16] Gareth Loy and Alexander Zelinsky, "Fast Radial Symmetry for Detecting Points of Interest," IEEE Transactions on Pattern Analysis and Machine Intelligence, vol. 25, no. 8, Aug. 2003.

[17] L. Dreschler and H. Nagel, "Volumetric model and 3-D trajectory of a moving car derived from monocular TVframe sequence of a streetscene," in Proc. IJCAI, 1981, pp. 692-697.

[18] M. Shah and R. Jain, "Detecting time-varying corners," Comput. Vision, Graph., Image Processing, vol. 28, no. 3, pp. 345-355, Dec. 1984.

[19] L. Kitchen and A. Rosenfeld, "Gray-level corner detection," Pattern Recognit. Lett., vol. 1, pp. 95-102, 1982.

[20] O. A. Zuniga and R. Haralik, "Corner detection using the facet model," in Proc. IEEE CVPR Conf., 1983, pp. 3037.

[21] K. Rangarajan, M. Shah, and D. Van Brackle, "Optimal corner detector," Comput. Vision, Graph., Image Processing, vol. 48, no. 2, pp. 230-245,Nov. 1989.

[22] A. de la Escalera, J.M armingol, M.A. Salichs Systems Enginnering and Automation Division, Universidad Carlos III de Madrid, Leganés, Madrid, Spain. Phone: +916249430, Fax: +916249431, E-mail: escalera@ing.uc3m.es

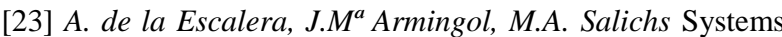
Enginnering and Automation Division, Universidad Carlos III de Madrid, Leganés, Madrid, Spain. Phone: 
+916249430, Fax: +916249431, E-mail: escalera@ing.uc3m.es

[24] David G. Lowe, iObject Recognition from Local Scale Invariant Featuresî, ICCV 1999, pp. 1150- 1157, 1999.

[25] J. Torresen, J. W. Bakke, and L. Sekania. Efficient recognition of speed limit signs. In Proc. IEEE Conf. on Intelligent Transportation Systems (ITS), Washington, DC, 2004.

[26] P. Viola and M. J. Jones. Robust real-time object detection. Technical Report CRL 2001/01, Cambridge Research Laboratory, 2001.

[27] B. Xie, D. Comaniciu, V. Ramesh, T. Boult, and M. Simon.Component fusion for face detection in the presence of heteroscedastic noise. In 25th Pattern Recognition Springer Verlag.
[28] M. M. Zadeh, T. Kasvand, and C. Y. Suen. Localization and recognition of traffic signs for automated vehicle control systems. In Proc. SPIE Vol. 3207, Intelligent Transportation Systems, pages 272-282, 1998.

[29] Y. Zhu, D. Comaniciu, M. Pellkofer, and T. Koehler. An integrated framework of vision-based vehicle detection with knowledge fusion. In IEEE Intelligent Vehicles Symposium (IV), Las Vegas, NV, 2005.

[30] M. de Saint Blancard. "Road sign recognition: A study of vision-based decision making for road environment recognition." In Vision-based Vehicle Guidance, Springer Series in Perception Engineering. SpringerVerlag, 1992. 